\title{
Adeno-associated virus-mediated survivin mutant Thr34Ala cooperates with oxaliplatin to inhibit tumor growth and angiogenesis in colon cancer
}

\author{
ZHIMOU XUE ${ }^{1}$, PING HU SUN ${ }^{2}$, LI MING ZHU ${ }^{2}$, SHI HU JIANG ${ }^{2}$, \\ MIN MIN QIAO ${ }^{2}$, ALFRED L. $\mathrm{CHI}^{3}$ and SHUI PING TU ${ }^{2}$ \\ ${ }^{1}$ Laboratory Animal Center of Soochow University, 199 Renai Road, Suzhou Industrial Park, Suzhou 215123; \\ ${ }^{2}$ Department of Gastroenterology, Rui-jin Hospital, Shanghai Jiaotong University School of Medicine, \\ Shanghai 200025, P.R. China; ${ }^{3}$ CHI Scientific, Inc., 63 Great Road, Maynard, MA 01754, USA
}

Received November 8, 2010; Accepted December 23, 2010

DOI: $10.3892 /$ or.2011.1166

\begin{abstract}
Colon cancer is one of the most common cancers. Survivin is overexpressed in human colon cancer and correlate with chemoresistance, angiogenesis and poor prognosis. Oxaliplatin, a platinum derivative cancer drug, has been used for treating human colorectal cancers. In the present study, we investigated the effect of the adeno-associated virus (AAV)mediated survivin mutant Thr34Ala [rAAV-Sur-Mut(T34A)] on colon cancer growth. Infection with rAAV-Sur-Mut(T34A) inhibited cell proliferation, induced apoptosis and mitotic catastrophe, and sensitized colon cancer cells to chemotherapeutic drugs in vitro. Treatment with rAAV-Sur-Mut(T34A) significantly induced apoptosis, reduced angiogenesis and inhibited colon cancer growth in vivo. More importantly, rAAV-Sur-Mut(T34A) treatment strongly enhanced the antitumor activity of oxaliplatin and prolonged animal survival. Thus, the use of rAAV-Sur-Mut(T34A) in combination with chemotherapy may be a promising strategy for colon cancer therapy.
\end{abstract}

\section{Introduction}

Colorectal cancer is one of leading causes of cancer-related deaths in the Western world. Although significant advances have occurred in the treatment of advanced colorectal cancers with the introduction of novel chemotherapies and targeted agents, the overall survival rate remains low, as advanced cancers eventually develop resistance to standard treatments through dysregulation of apoptosis (1-3). Survivin, a structurally unique member of inhibitor of apoptosis family (1-3),

Correspondence to: Dr Shui Ping Tu, Department of Gastroenterology, Rui-jin Hospital, Shanghai 200025, P.R. China

E-mail: tushuiping@yahoo.com

Key words: survivin mutant T34A, adeno-associated virus, colon cancer, apoptosis, mitotic catastrophe, angiogenesis is overexpressedin the most common cancers, including colon cancer (2,4-6). Adenoma-carcinoma sequence has been established in colorectal carcinogenesis $(7,8)$. A previous study demonstrated that survivin expression increased in the transition from adenoma with low dysplasia to high dysplasia/ carcinoma, which was associated with a decrease in tumor cell apoptosis and increase in cell proliferation and angiogenesis during colorectal tumorigenesis (9). The development of colorectal carcinoma proceeds through a series of genetic alterations involving the loss of tumor suppressor and activation of oncogenes genes (e.g., APC, p53, DCC and ras) (10). Studies have shown that p53 and APC suppressed survivin expression $(11,12)$. Colorectal cancer frequently displays $A P C$ and p53 gene mutation, thus p53 and APC mutant contribute to early colonic tumorigenesis by allowing constitutive expression of survivin, which ultimately prevents apoptosis and results in cellular immortality $(12,13)$. Furthermore, surivin expression is correlated with shorter survival rate in patients with colorectal cancer $(5,14)$. Therefore, targeting inhibition of survivin expression and function may be a new strategy for colon cancer therapy.

It has been demonstrated that phosphorylation of survivin on Thr34 site controls survivin stability and/or ubiquitindependent degradation $(15,16)$. Ectopic overexpression of non-phosphorylatable survivin mutant (T34A) may abolish kinase $\mathrm{p} 34^{\mathrm{cdc} 2}$-cyclin $\mathrm{B} 1$ on the survivin phosphorylation, resulting in the ubiquitin-dependent degradation of survivin (17). Previous studies have shown that plasmids or a replication-deficient adenovirus mediated survivin mutant T34A could induce apoptosis and inhibite tumor growth in vivo $(18,19)$, indicating that survivin mutant T34A is a promising target for cancer therapy. However, the transfer of plasmid DNA is typically an inefficient process, and adenoviralmediated gene transfer is complicated by a host immune response to the transduced target cells (20). Adeno-associated virus (AAV) is a small virus that does not cause currently known human disease and immune response (21). Furthermore, AAV vectors penetrate human solid tumor tissue in vivo more effectively than adenoviral vectors (13). Thus, AAV is becoming a very attractive candidate for creating viral vectors for gene therapy. 
Oxaliplatin, a recently developed third-generation cisplatin analogue, has displayed clinical activity in a wide variety of tumor types. Synergistic with 5-FU in colorectal cancer $(\mathrm{CRC})$, the combination has proven efficacy in 5-FU-resistant advanced disease and in previously untreated CRC. Oxaliplatin is an important new anticancer agent. In our previous study, we demonstrated that AAV-Sur-Mut(Cys84Ala) significantly enhanced the antitumor effect of 5-FU, resulted in tumor regression (22). However, the effect of AAV-mediated survivin mutant T34A alone or combination with oxaliplatin on human colon cancer growth has not been investigated. In the present study, we have demonstrated for the first time that AAV-mediated-survivin-Mut(Thr34Ala) induced apoptosis, inhibited angiogenesis and tumor growth in colon cancer, and cooperated with oxaliplatin-induced tumor regression.

\section{Materials and methods}

Cell culture and chemicals. Human colon cancer cell lines HCT-116 and Colo 205 (ATCC, Rockville, MD, USA) were maintained in RPMI-1640 containing $10 \%$ fetal bovine serum (FBS), $100 \mathrm{U} / \mathrm{ml}$ penicillin, $100 \mu \mathrm{g} / \mathrm{ml}$ streptomycin (Gibco BRL, Life Technologies, NY, USA). Oxaliplatin $(5 \mu \mathrm{g} / \mathrm{ml})$ (Pharmacia and Upjohn Limited Corp., Australia) was solubilized in sterilized water and stored at $4^{\circ} \mathrm{C}$.

Construction of survivin dominant-negative mutant plasmids. We used reverse-transcriptase polymerase chain reaction (RT-PCR) and an overlap extension PCR to construct pcDNA3-survivin and pcDNA3-dominant-negative mutant (T34A) plasmids as previously described (23). The T34A mutation was introduced by site-directed mutagenesis into pcDNA3-survivin cDNA by using the oligonucleotide 5'-GG CTGCGCCTGCgCCCCGGAGCGGATG-3'. All of the constructs were confirmed by sequencing.

Construction and generation of recombinant $A A V$. We constructed 3 recombinant AAV type plasmids encoding survivin, survivin mutant (T34A) and EGFP, respectively (22). Briefly, full-length $\operatorname{Sur}(w t)$ and Sur-Mut(T34A) cDNAs were cut with BamHI and XhoI from pcDNA3-Survivin and pcDNA3-SurMut(T34A), respectively, and subcloned into the corresponding BamHI and XhoI sites of pAM/CAG-WPRE-BGHpolyA to generate pAM/CAG-Sur-Mut(T34A). Recombinant AAV virus stocks were generated and purified by HiTrap Heparin column chromatography (Sigma Chemical Co., St. Louis, MO). The AAV viral genome titer was quantified by real-time PCR using TaqMan (Perkin-Elmer Biosystems, Foster City, CA). The viral vector was stored at $-80^{\circ} \mathrm{C}$ before experiments.

Flow cytometry analysis. Cells were collected and fixed in ice-cold $70 \%$ ethanol in PBS and stored at $-20^{\circ} \mathrm{C}$ before use. After resuspension, cells were washed and incubated with $100 \mu \mathrm{l}$ of RNase I $(1 \mathrm{mg} / \mathrm{ml})$ and $100 \mu 1$ of propidium iodide (400 $\mu \mathrm{g} / \mathrm{ml}$; Sigma) at $37^{\circ} \mathrm{C}$ for $30 \mathrm{~min}$. Samples were analyzed by flow cytometry (Coulter, Luton). The cell cycle phase distribution was calculated from the resultant DNA histogram using Multi-cycle AV software (Phoenix Flow Systems, San Diego, CA). Cells with subdiploid DNA content were considered apoptotic cells.
Western blot analysis. Cells were lysed in lysis buffer (22). Proteins were electrophoresed on $10 \%$ denaturing sodium dodecylsulfate gel, and transferred to Immobilon-P membrane (Millipore, Bedford, MA). The blots were incubated with specific primary antibodies, reacted with a peroxidaseconjugated second antibody (Santa Cruz, CA) and then visualized by enhanced chemiluminescence (ECL, Amersham, Piscataway, NJ). Rabbit Survivin (71G4B7) monoclonal antibodies were purchased from Cell Signaling (Danvers, MA), Rabbit XIAP monoclonal antibody $(2 \mu \mathrm{g} / \mathrm{ml})$ from StressGen Biothechnologies Corp. (Victoria, BC, Canada); p-Survivin (Thr34)-R antibody, caspase 3/9, cytochrome c and B-actin monoclonal antibodies were all from Santa Cruz Biotechnology (Santa Cruz, CA).

Immunofluorescence staining. Cells were fixed in $2 \%$ formaldehyde for $10 \mathrm{~min}$ and permeabilized with $0.5 \%$ Nonidet P40 in PBS. Antibodies to $\alpha$-tubulin (clone DM1A) and FITC-conjugated goat anti-mouse antibodies were purchased from Sigma. Antibodies were used at 1:100 dilutions for tubulins. Nuclei were stained with $1 \mu \mathrm{g} / \mathrm{ml}$ Hoechst 22358 and cells were analyzed using a Zeiss Axioscop fluorescence microscope (23).

In situ detection of apoptotic cells by TUNEL assay. Apoptosis in xenograft tumors was determined by TUNEL staining (Zymed, San Francisco, CA) according to production manuals. The percentage of apoptotic cells was assessed in 10 randomly selected fields viewed at $\mathrm{x} 400$ magnification. The apoptotic index (A/I) was calculated as number of apoptotic cells/total number of nucleated cells $\times 100 \%$.

In vivo colon cancer xenograft experiment. Five to 6-weekold female BALB/c athymic nude mice were obtained from Shanghai Experimental Animals Centre of Chinese Academy of Sciences. All animal studies were conducted under approved guidelines of the Animal Care and Use Committee of Shanghai Jiaotong University. Mice were injected subcutaneously on the flanks with $1 \times 10^{6}$ exponentially growing HCT-116 cells. Tumors were allowed to grow to $100-150 \mathrm{~mm}^{3}$ (5-7 mm diameter). Injection was given to 3 tumor sites with rAAVSur-Mut(T34A), rAAV-Sur(wt) or rAAV-EGFP at $5 \times 10^{10}$ viral particles/site of injection or with PBS on 3 consecutive days. Alternatively, mice were intraperitoneally (IP) injected with $5 \mathrm{mg} / \mathrm{kg}$ oxaliplatin or a combination of rAAV virus and oxaliplatin for 3 days. Tumor growth was measured weekly after injection. Tumor volumes were calculated using the following formula: $\mathrm{V}\left(\mathrm{mm}^{3}\right)=0.52 \mathrm{x}$ length $(\mathrm{mm}) \mathrm{x}$ width $(\mathrm{mm})^{2}(22,23)$.

Immunohistochemistry staining. Coloncancer xenograft tissues were harvested after 7 days of treatment, formalinfixed and paraffin-embedded. Tumor sections were suffered antigen retrieval in $10 \mathrm{mM}$ citrate buffer ( $\mathrm{pH} \mathrm{6}$; Bio Genex, San Ranmon, CA) and then incubated with CD31 (PECAM-1) polyclonal antibody (1:50; PharMingen, San Diego, CA), followed by biotinylated anti-IgG antibody (1:200; Dako) and streptavidin-biotinylated-complex/horseradish peroxidase (Dako). The sections were counterstained with hematoxylin. MVD was evaluated according to the method described 
previously (23). Areas of the highest neovascularization were chosen, and microvessel counting was performed at x200 magnification in three chosen fields. Any immunoreactive endothelial cell or endothelial cell cluster that had been distinctly separated from adjacent microvessels was considered a single countable vessel. The results regarding angiogenesis in each tumor were expressed as the absolute number of vessels/ $0.74 \mathrm{~mm}^{2}$ (x200 field). In all assays, matched isotype control antibodies were used and found to be unreactive in all cases.

Statistical analysis. Data are expressed as means of at least 3 different experiments \pm SD (standard derivations). The results were analyzed by Student's t-test or Mann-Whitney test. P-value $<0.05$ was considered statistically significant. The Kaplan-Meier method was used to analyze survival of tumor-bearing mice.

\section{Results}

rAAV-Sur-Mut(T34A) virus induces apoptosis in colon cancer cells. Our previous study showed that AAV can efficiently transduce colon cancer cells (22). To investigate the effect of survivin-Mut (T34A) on colon cancer growth, we generated AAV-mediated survivin mutant (T34A) virus [AAV-Sur-Mut(T34A)]. The HCT-116 colon cancer cells transduced with the AAV-Sur-Mut(T34A) and AAV-Sur(wt) virus efficiently expressed survivin protein (increased survivin expression assumed to reflect the cumulative levels of endogenous wild-type survivin and overexpressed dominantnegative mutant survivin) (Fig. 1A, upper lane). Furthermore, the expression of survivin mutant by rAAV-Sur-Mut(T34A) virus significantly down-regulated the level of endogenous Thr34-phosphorylated survivin as detected by Western blot analysis using antibody against phosphorylated Thr34 (p-Survivin 34) (Fig. 1A, middle lane) in colon cancer HCT-116 cells, confirming that rAAV-Sur-Mut(T34A) virus efficiently transduced survivin mutant (T34A) expression.

To characterize further the pro-apoptotic effect of $\mathrm{rAAV}$ Sur-Mut(T34A), we analyzed apoptosis induced by transduction with rAAV-Sur-Mut(T34A), rAAV-Sur(wt) and rAAV-EGFP. Transduction of rAAV-Sur-Mut(T34A) induced apoptosis in HCT-116 and Colo 205 cell lines (Fig. 1B). Transduction of rAAV-Sur-Mut(T34A) consistently resulted in expression of mutant survivin protein and several markers of apoptosis, such as caspase 3/9 and PARP cleavage, and the release of mitochondrial cytochrome $\mathrm{c}$ into the cytosol (Fig. 1C). Survivin expression levels detected by immunoblotting were assumed to reflect the cumulative levels of endogenous wild-type survivin and overexpressed dominantnegative mutant survivin (Fig. 1C). In contrast, there was no significant change in expression of other IAP genes (XIAP, cIAP1, cIAP2) in cells transfected with rAAV-Sur-Mut(T34A) (data not shown).

rAAV-Sur-Mut(T34A) induces mitotic catastrophe in colon cancer cells. Ours and other studies have indicated that the inhibition of survivin function caused mitotic catastrophe (23). We examined whether transduction of AAV-SurMut(T34A) could induce mitotic catastrophe in colon cancer cells. We found that infection of AAV-Sur-Mut(T34A)
A

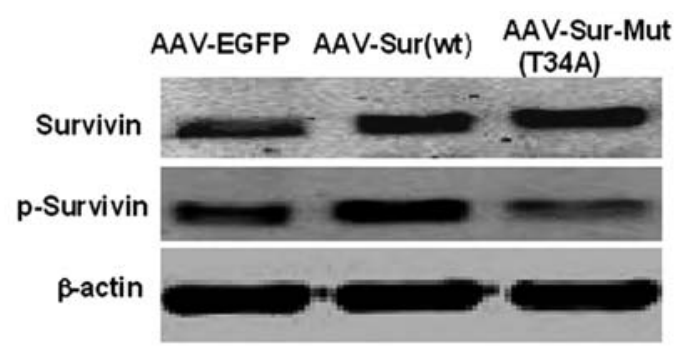

B
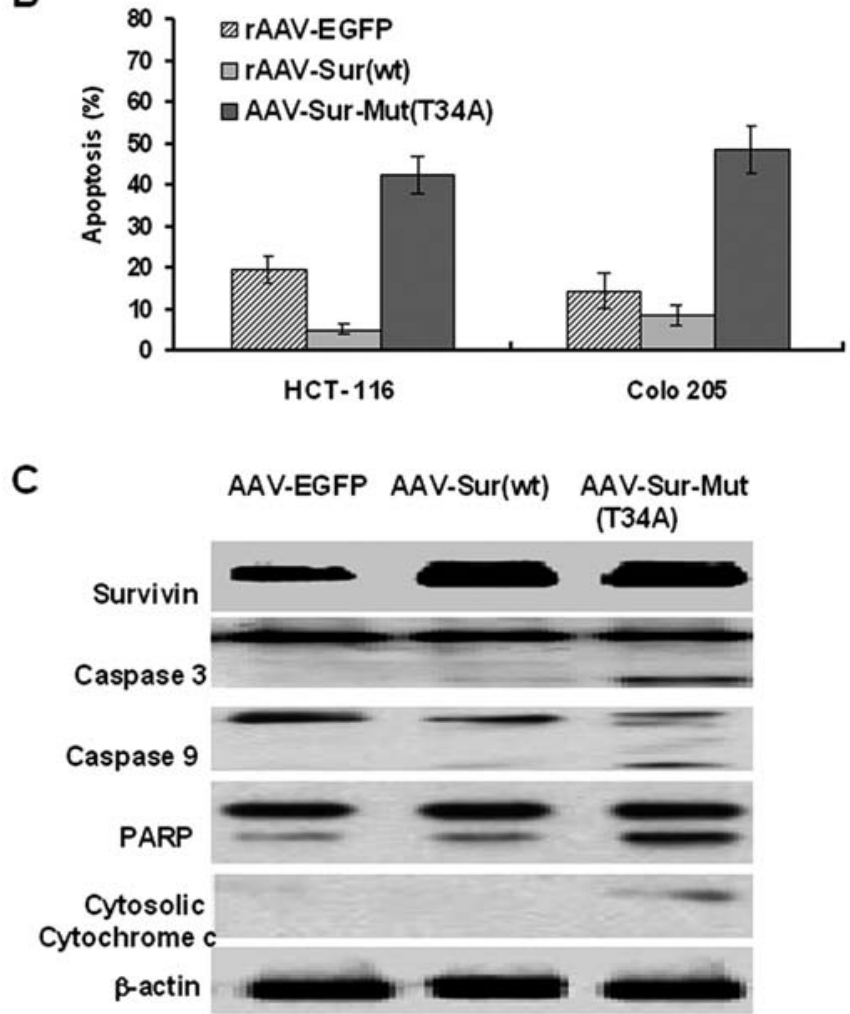

Figure 1. rAAV-Sur-Mut(T34A) induces apoptosis in colon cancer cells. (A) Transduction of rAAV-Sur-Mut(T34A) virus inhibit expression of endogenous p-Survivin. Total survivin and p-Survivin were detected by Western blot analysis. (B) rAAV-Sur-Mut(T34A) induces apoptosis in colon cancer cells. Cells were infected with rAAV at $1 \times 10^{5}$ viral particles/cell for $48 \mathrm{~h}$. Apoptotic cells were analyzed by FACS. The results represent the means \pm SEM from 3 independent experiments. ${ }^{*} \mathrm{P}<0.05$, compared to rAAV-EGFPtreated group. (C) Infection of rAAV-Sur-Mut(T34A) induced expression of mutant survivin protein, caspase 3 and PARP cleavage, and released mitochondrial cytochrome $\mathrm{c}$ in HCT-116 cells. Proteins were detected by Western blot analysis.

increased the number of cells with multiple nuclei in HCT-116 cells (Fig. 2A). Consistent with these observations, cells that were transduced with AAV-Sur-Mut(T34A) showed a significant increase in abnormal nuclei, including micronucleated, multilobulated and abnormally large nuclei $72 \mathrm{~h}$ after transduction, relative to cells transduced with rAAV-Sur(wt) and rAAV-EGFP (Fig. 2B). Our results show that the rAAVmediated survivin mutant (T34A) causes mitotic catastrophe in colon cancer cells.

rAAV-Sur-Mut(T34A) virus sensitizes colon cancer cells to oxaliplatin-induced apoptosis. 5-Fluorouracil (5-FU) is the first-choice chemotherapy drug for colorectal cancer. Oxaliplatin is used for the treatment of advanced colorectal 
A

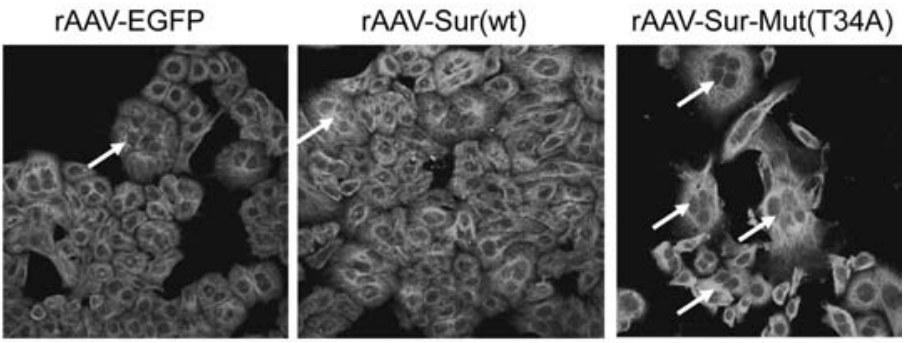

B

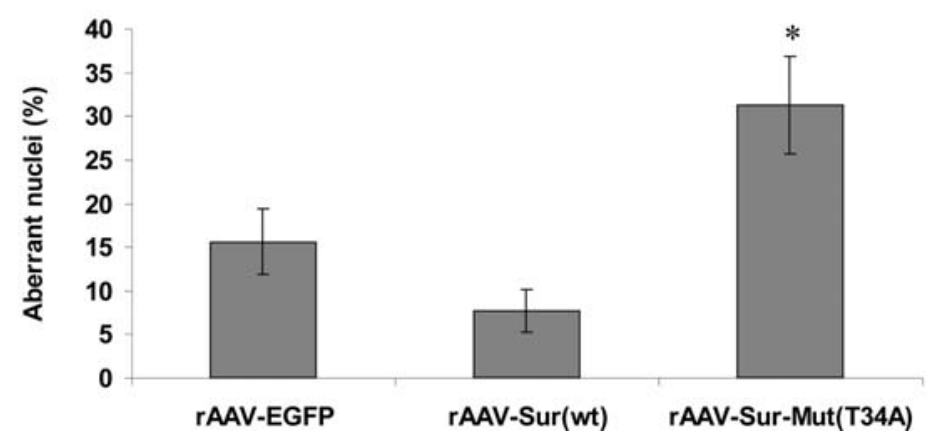

Figure 2. rAAV-Sur-Mut(T34A) Virus transduction induces mitotic catastrophe in colon cancer cells. (A) HCT-116 cells transduced with rAAV-Sur(wt), rAAV-EGFP or rAAV-Sur-Mut(T34A) for $72 \mathrm{~h}$ were stained for microtubules with an anti-r-tubulin antibody. Arrow shows abnormal large and multilobed nuclei. Photomicrographs are from representative experiments performed in triplicate. Original magnification, $\mathrm{x} 400$. (B) Quantification of mitotic catastrophe. Approximately 500-600 nuclei were scored on 5 random $400 \mathrm{x}$ objective fields in triplicate as described. The experiment was performed independently, and the results presented are the means \pm SD obtained from 3 independent experiments. ${ }^{*} \mathrm{P}<0.01$ compared with group transduced with rAAV-Sur(wt) and rAAV-EGFP.

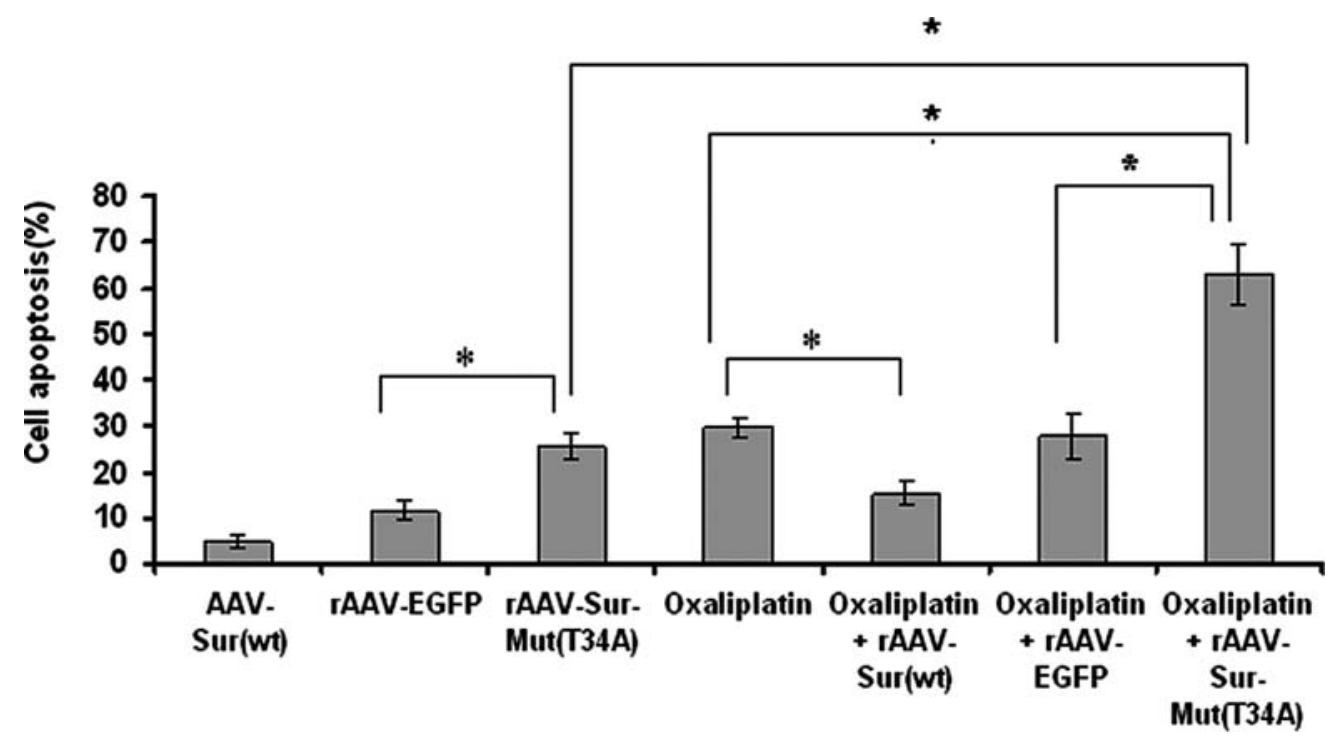

Figure 3. rAAV-Sur-Mut(T34A) virus sensitizes colon cancer cells to oxaliplatin-induced apoptosis. HCT-116 cells were infected with rAAV at $1 \times 10^{5}$ viral particle/cell followed by $1 \mu \mathrm{g} / \mathrm{ml}$ of oxaliplatin for an additional $72 \mathrm{~h}$. Apoptosis was determined by FACS analysis. The results represent the mean \pm SD of 3 independent experiments. ${ }^{*} \mathrm{P}<0.05$, compared to oxalipatin treatment alone; ${ }^{\#} \mathrm{P}<0.05$, compared to other treatment groups, respectively.

cancer that is resistant to 5-FU (24). Ectopic expression of survivin increased the resistance of colon cancer to oxaliplatin. Thus, we asked whether ectopic expression of survivn-Mut (T34A) could sensitize colon cancer to apoptosis induced by oxaliplatin. We examined the pro-apoptotic effect of rAAV-Sur-Mut(T34A) combined with the agents on cancer cells. The results showed that the rate of apoptosis was increased $>2.5$-fold when a combination of oxaliplatin and
rAAV-Sur-Mut(T34A) virus was used relative to either treatment group alone (Fig. 3). In contrast, transduction with rAAV-Sur(wt) counteracted apoptosis induced oxaliplatin. These results demonstrate that rAAV-Sur-Mut(T34A) sensitizes colon cancer cells to chemotherapeutic drugs.

rAAV-Sur-Mut(T34A) inhibits tumor growth in vivo. We examined the effect of rAAV-Sur-Mut(T34A) on subcutaneous 
A

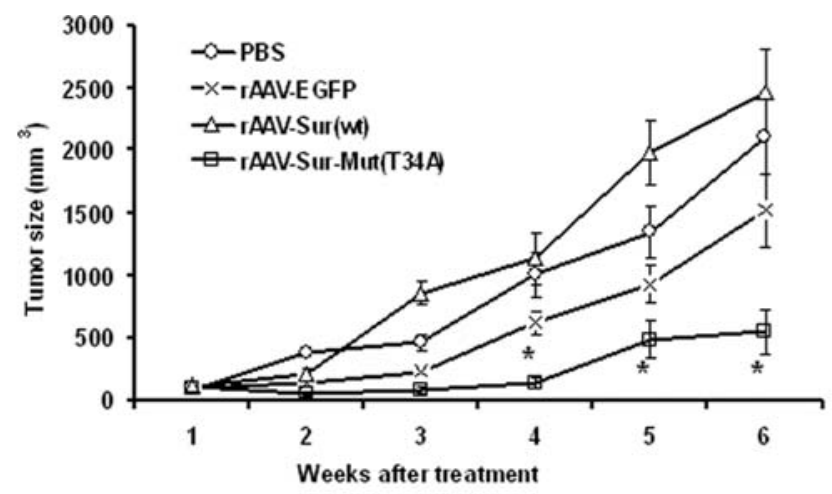

B
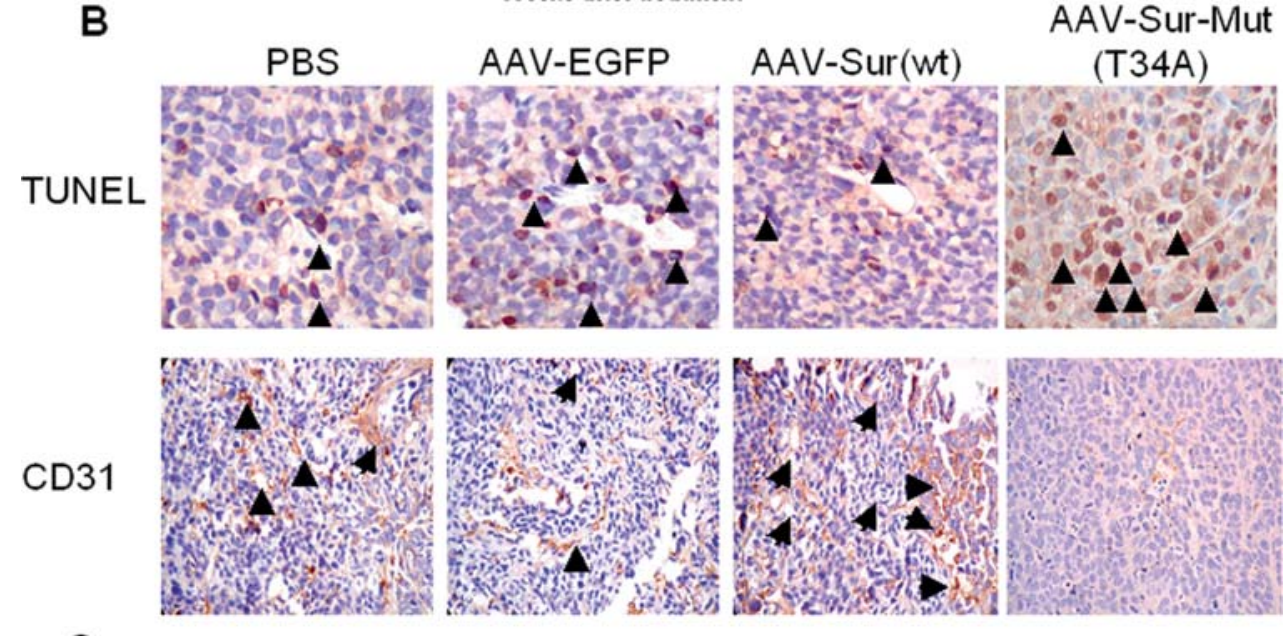

C
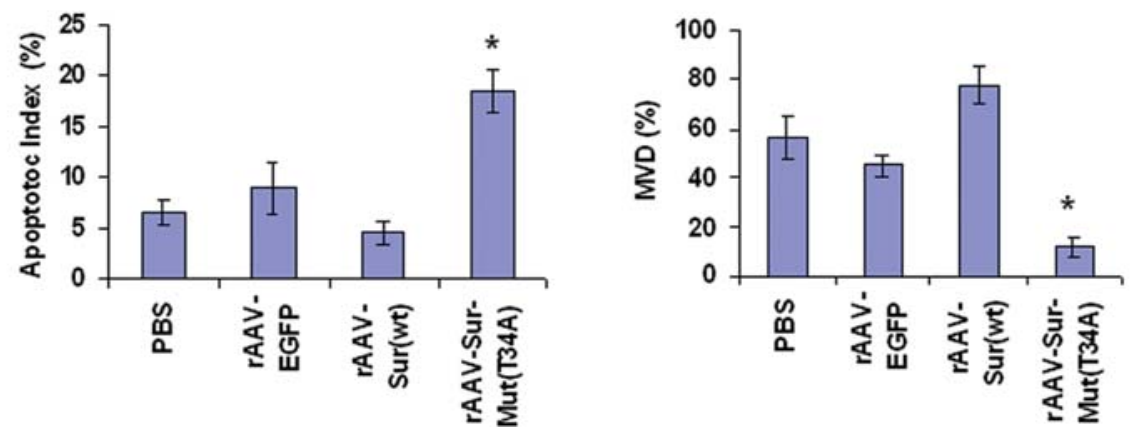

Figure 4. rAAV-Sur-Mut(T34A) inhibits tumor growth in vivo. (A) The HCT-116 cells were subcutaneously injected into the right flank of athymic female nude mice. Tumors with $100-150 \mathrm{~mm}^{3}$ volume were injected in 3 sites with indicated rAAV virus at $5 \times 10^{10}$ viral particles/site of injection or with PBS. Tumor growth was measured every week after injection. Data are the means \pm SD of tumor size per mouse. (B) AAV-mediated survivin mutant (T34A) expression induces apoptosis and inhibits tumor angiogenesis in vivo. Tumor sections from TUNEL staining for detection of apoptotic cells or immuno-histochemical stained for CD31 (angiogenesis) (original magnification, x200). ${ }^{*} \mathrm{P}<0.05$. Arrows show apoptotic cells (upper lane) and CD31-positive cells (lower lane), respectively. (C) Quantification of apoptosis of tumor cells. TUNEL staining is shown (B). The number of apoptotic cells was assessed in 10 randomly selected fields of each slide viewed at x40 magnification. Data are the mean \pm SD of the apoptotic index from 9 sections of 3 animals per group. (D) Quantification of blood microvessel density (BMD). CD31 staining is shown (B). Areas of highest vascularization were chosen at low magnification, $\mathrm{x} 100$ ). Microvessel counting was performed at x200 on three chosen fields. Results are the mean of independent determinations by two investigators. Data represent the mean $\pm \operatorname{SD}(n=5)$.

colon cancer xenograft tumors. The HCT-116 cells were injected into the flank of nude mice, and tumors were allowed to reach a volume of $\sim 100-150 \mathrm{~mm}^{3}$ over a 7 - to 10 -day period (5-7 mm diameter). The intratumor administration of rAAVSur-Mut(T34A) inhibited HCT-116 xenograft growth by $\sim 75.3 \%$ at 5 weeks post-injection, whereas treatment with rAAV-EGFP only reduced tumor growth by $34.2 \%$ (Fig. 4A). The intratumor administration of rAAV-Sur(wt) increased tumor growth by $\sim 16.4 \%$ at 4 weeks, compared with groups injected with PBS (Fig. 4A). Similar results were obtained in
HT-29 cells xenograft. Single intratumor administration of rAAV-Sur-Mut(T34A), rAAV-EGFP, inhibited HT-29 xenograft growth by a $\sim 58.7$ and $33.4 \%$ at 5 weeks, compared with groups injected with PBS. These results showed that single intratumor administration of rAAV-Sur(wt) significantly inhibited colon cancer growth.

rAAV-Sur-Mut(T34A) induces apoptosis and inhibits tumor angiogenesis in vivo. We further investigated the in vivo mechanism by which rAAV-Sur-Mut(T34A) inhibits tumor 

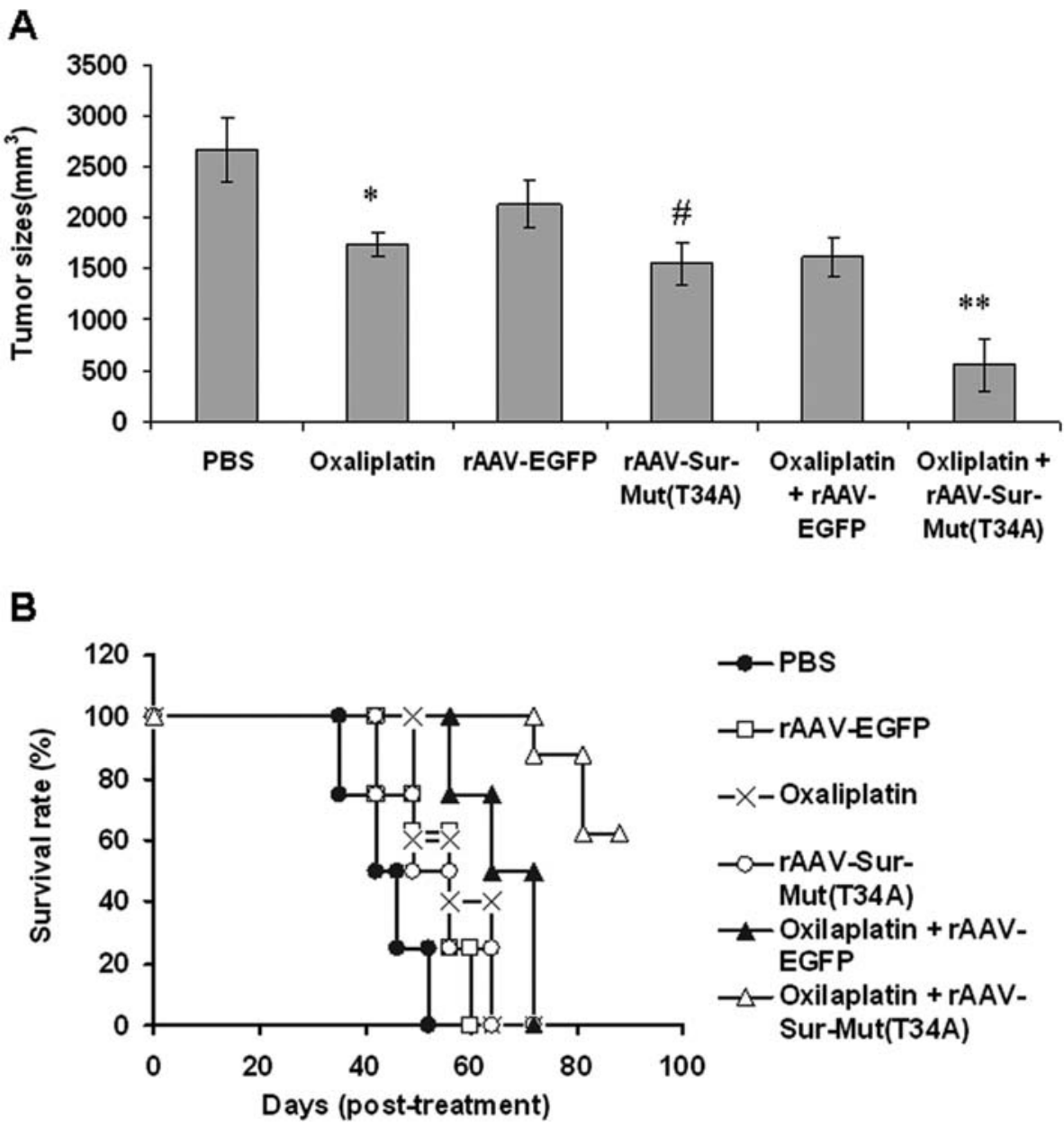

Figure 5. The combination of rAAV-Sur-Mut(T34A) oxaliplatin to enhances antitumor in vivo. (A) The combination of rAAV-Sur-Mut(T34A) and oxaliplatin synergistically inhibits tumor growth. Tumors $\sim 100-150 \mathrm{~mm}^{3}$ volume was injected with PBS, rAAV-EGFP, or rAAV-Sur(wt) or rAAV-Sur-Mut in 3 sites $\left(5 \times 10^{10}\right.$ particles virus/site) in combinations of oxalipatin injection $(5 \mathrm{mg} / \mathrm{kg})$. The experiments were repeated twice. Tumor growth was measured every week after injection. Data are the means $\pm \mathrm{SD}$ of tumor size per mouse 5 weeks after treatment. ${ }^{*} \mathrm{P}<0.05$, compared to PBS group; ${ }^{\#} \mathrm{P}<0.05$, compared to rAAV-EGFP group; ${ }^{* *} \mathrm{P}<0.05$, compared to other treatment groups. (B) The combination of rAAV-Sur-Mut(T34A) and oxaliplatin prolongs animal survival. The experimental conditions were the same as in (A). Survival was monitored every day, and tumor volume was measured every week after treatment. Definition of death is natural death because of tumor burden or sacrificed because of tumor sizes (diameter) $>2.5 \mathrm{~cm}(\mathrm{n}=10)$.

growth. Tumors injected with rAAV-Sur-Mut(A34T) virus expressed high levels of survivin mutant protein (data not shown) 42 days after injection of rAAV virus. Consistent with the expression of survivin mutant protein, TUNEL-positive cells (apoptotic cells) were significantly higher in the tumors injected with rAAV-Sur-Mut(T34A) virus compared to other tumors injected with PBS, rAAV-EGFP and rAAV-Sur(wt) (Fig. 4B). These results indicated that rAAV-Sur-Mut(T34A) virus may mediate long-term expression of survivin mutant and induce apoptosis in colon cancer in vivo.

It has been reported that survivin could promote capillary formation in vitro $(25,26)$. We examined whether overexpression of survivin mutant (T34A) could inhibit tumor angiogenesis in colon cancer. Immunohistochemical staining of endothelial cell-derived CD31 in colon cancer xenografts showed that the tumors injected with rAAV-Sur(wt) and AAV-GFP exhibited an extensive network of viable blood vessels, consistent with tumor-associated angiogenesis. In contrast, intratumor injection of rAAV-Sur-Mut(T34A) resulted in a significant reduction in the number of viable blood vessels by CD31 staining (Fig. 5B). Quantification analysis of
CD31-stained areas showed injection of AAV-Sur-Mut(T34A) in tumor resulted in significant suppression of blood vessel density in colon cancer xenografts, when compared with AdAAV-Sur(wt), AAV-EGFP and PBS (Fig. 4C).

rAAV-Sur-Mut(T34A) enhances the effect of antitumor chemotherapeutic drugs in vivo. We further investigated the combination effect of rAAV-Sur-Mut(T34A) and oxalipatin on tumor growth. These tumors were injected in 3 sites with rAAV-Sur(wt), rAAV-EGFP or rAAV-Sur-Mut(T34A) at $5 \times 10^{10} \mathrm{pfu} / \mathrm{site}$ and together with $10 \mathrm{mg} / \mathrm{kg} /$ dose of oxaliplatin for 5 days. rAAV-Sur-Mut(T34A) enhanced the antitumor activity of oxaliplatin. In contrast, cotreatment with rAAVEGFP did not potentiate the antitumor effect of oxaliplatin 5 weeks post-treatment (Fig. 5A). We further extended the observation on the effect of co-treatment of rAAV-SurMut(T34A) and oxaliplatin on the long-term survival of mice bearing tumor. Mice challenged with HCT-116 cells died from tumor burden or were sacrified when they reached experimental end-points (tumor size $2.5 \mathrm{~cm}^{3}$ ). Although mice treated with oxalipatin or rAAV-Sur-Mut(T34A) alone 
survived significantly longer than PBS-treated mice $(\mathrm{P}<0.05)$, all eventually died (Fig. 5B). Notably, 5 out of 8 animals treated with the combination of rAAV-Sur-Mut(T34A) and oxaliplatin were still alive without any visible tumors and without clinical symptoms 100 days after treatment. Furthermore, all the animals survived significantly longer than mice treated with rAAV-Sur-Mut(T34A) or oxaliplatin $(\mathrm{P}<0.01)$ (Fig. 5B).

\section{Discussion}

Survivin expression is associated with the inhibition of apoptosis and poor progression in cancer patients. Thus, targeting survivin pathway for cancer therapy has been widely investigated $(18,25,27-29)$. The studies have shown that the mutagenesis of $\mathrm{Thr}^{34} \rightarrow$ Ala completely suppressed survivin phosphorylation by $\mathrm{p} 34^{\text {cdc2 }}$-cyclin $\mathrm{B} 1$ in vitro and in vivo $(17,30)$. The inducible expression (28) or adenoviral delivery (18) of nonphosphorylatable survivin mutant (T34A) prevented phosphorylation of endogenous survivin, induced apoptosis and inhibited tumor growth in human breast and prostate cancer and mouse colon cancer in vivo $(18,19,28,31,32)$. In this study, we demonstrated that AAV-mediated survivin-Mut (T34A) induced apoptosis of human colon cancer in vitro and in vivo, reduced angiogenesis and inhibited colon cancer growth in vivo. The treatment of rAAV-Sur-Mut(T34A) virus strongly enhanced the antitumor activity of oxaliplatin and prolonged animal survival.

Tumor angiogenesis plays an important role in tumor development and progression. Several studies have shown that the elevated survivin expression is associated with increased microvessel density in colorectal cancer (9), colon cancer (23) and brain glioma (33). Increased survivin expression has been verified in endothelial cells during the proliferative $(25,34,35)$ and remodeling $(36,37)$ phases of angiogenesis, potentially serving as a cytoprotective mechanism for these cells (35). Importantly, induction of survivin in endothelial cells is associated with resistance to apoptosis. In contrast, survivin is undetectable in quiescent endothelium in vitro and in vivo (25), and targeting survivin does not affect endothelial cell viability in quiescent endothelium (33). Thus, inhibition of survivin may promote endothelial cell apoptosis during tumor angiogenesis, accelerating regression of newly formed blood vessels and reducing the incidence of metastatic disease. In this study, we found that rAAV-mediated SurMut(T34A) could markedly suppress tumor angiogenesis in vivo. This was consistent with our previous results that rAAV-mediated Sur-mut(Cys84Ala) induced endothelial cell apoptosis and reduced angiogenesis in colon cancer (22). Our data showed that the selected expression of survivin dominative mutant in tumor cells may provide a high degree of specificity for potential survivin antagonists to enhance both anti-angiogenic and anti-neoplastic therapeutic strategies.

Chemotherapy is still one of major strategies for advanced colon cancer therapy. However, drugs resistance is still a potential clinical problem. It has been demonstrated that overexpression of survivin contributes to drug-resistance $(37,38)$ and that anticancer drugs inducing G2-M arrest with elevated or residual p34 ${ }^{\text {cdc2 }}$ kinase activity caused Thr34 phosphorylation and increased survivin levels (27). 5-FU and oxaliplatin have been shown to induce $\mathrm{G} 2 / \mathrm{M}$ arrest in a variety of cell lines and induce apoptosis (39-41). In this study, overxpression of survivin mediated by rAAV virus reduced oxaliplatin-induced cells apoptosis, consistent with previous reports that the ectopic expression of survivin contributes to tumor-resistance of drugs $(27,42)$. Accordingly, the expression of Sur-Mut(T34A) mediated by AAV sensitized colon cancer cells to oxaliplatin (42). Furthermore, the combination of oxaliplatin and rAAV-mediated Sur-Mut(T34A) significantly inhibited tumor growth in vivo than rAAV-Sur-Mut(T34A) only injection and oxaliplatin only treatment. Our study demonstrated a synergistic effect of the combination of targeting survivin gene therapy and chemotherapy. Use of oxaliplatin as second-line therapy for advanced colorectal cancer has shown some promise in cases where 5-fluorouracil has failed. Thus, the findings described here may have practical implications for potential strategies for colon cancer therapy.

In summary, our study documented the therapeutic potential of rAAV mediated Sur-Mut(T34A) in the treatment of colon cancer, especially in combination with chemotherapeutic intervention. Our results showed that targeting the survivin pathway is a novel anti-angiogenic and anti-neoplastic therapeutic strategy.

\section{Acknowledgements}

This study was supported by the National Natural Science Foundation of China (30772518, to S.P.T.).

\section{References}

1. Adida C, Crotty PL, McGrath J, Berrebi D, Diebold J and Altieri DC: Developmentally regulated expression of the novel cancer anti-apoptosis gene survivin in human and mouse differentiation. Am J Pathol 152: 43-49, 1998.

2. Ambrosini G, Adida C and Altieri DC: A novel anti-apoptosis gene, survivin, expressed in cancer and lymphoma. Nat Med 3: 917-921, 1997.

3. Deveraux QL and Reed JC: IAP family proteins - suppressors of apoptosis. Genes Dev 13: 239-252, 1999

4. Li F, Ambrosini G, Chu EY, et al: Control of apoptosis and mitotic spindle checkpoint by survivin. Nature 396: 580-584, 1998.

5. Kawasaki H, Altieri DC, Lu CD, Toyoda M, Tenjo T and Tanigawa N: Inhibition of apoptosis by survivin predicts shorter survival rates in colorectal cancer. Cancer Res 58: 5071-5074, 1998.

6. Sarela AI, MacAdam RC, Farmery SM, Markham AF and Guillou PJ: Expression of the antiapoptosis gene, survivin, predicts death from recurrent colorectal carcinoma. Gut 46: 645-650, 2000

7. Bedi A, Pasricha PJ, Akhtar AJ, et al: Inhibition of apoptosis during development of colorectal cancer. Cancer Res 55: 1811-1816, 1995.

8. Sinicrope FA, Ruan SB, Cleary KR, Stephens LC, Lee JJ and Levin B: bcl-2 and p53 oncoprotein expression during colorectal tumorigenesis. Cancer Res 55: 237-241, 1995.

9. Kawasaki H, Toyoda M, Shinohara H, et al: Expression of survivin correlates with apoptosis, proliferation, and angiogenesis during human colorectal tumorigenesis. Cancer 91: 2026-2032, 2001 .

10. Chung DC: The genetic basis of colorectal cancer: insights into critical pathways of tumorigenesis. Gastroenterology 119: 854-865, 2000.

11. Zhang T, Otevrel T, Gao Z, Ehrlich SM, Fields JZ and Boman BM: Evidence that APC regulates survivin expression: a possible mechanism contributing to the stem cell origin of colon cancer. Cancer Res 61: 8664-8667, 2001.

12. Hoffman WH, Biade S, Zilfou JT, Chen J and Murphy M: Transcriptional repression of the anti-apoptotic survivin gene by wild-type p53. J Biol Chem 277: 3247-3257, 2002. 
13. Enger PO, Thorsen F, Lonning PE, Bjerkvig R and Hoover F: Adeno-associated viral vectors penetrate human solid tumor tissue in vivo more effectively than adenoviral vectors. Hum Gene Ther 13: 1115-1125, 2002.

14. Sarela AI, Scott N, Ramsdale J, Markham AF and Guillou PJ: Immunohistochemical detection of the anti-apoptosis protein survivin, predicts survival after curative resection of stage II colorectal carcinomas. Ann Surg Oncol 8: 305-310, 2001.

15. Zhao J, Tenev T, Martins LM, Downward J and Lemoine NR: The ubiquitin-proteasome pathway regulates survivin degradation in a cell cycle-dependent manner. J Cell Sci 113 Pt 23: 4363-4371, 2000.

16. Yang Y, Fang S, Jensen JP, Weissman AM and Ashwell JD: Ubiquitin protein ligase activity of IAPs and their degradation in proteasomes in response to apoptotic stimuli. Science 288: $874-877,2000$

17. O'Connor DS, Grossman D, Plescia J, et al: Regulation of apoptosis at cell division by p34cdc2 phosphorylation of survivin. Proc Natl Acad Sci USA 97: 13103-13107, 2000.

18. Mesri M, Wall NR, Li J, Kim RW and Altieri DC: Cancer gene therapy using a survivin mutant adenovirus. J Clin Invest 108: 981-990, 2001.

19. Li B, Fan J, Liu X, et al: Suppression of colorectal tumor growth by regulated survivin targeting. J Mol Med 84: 1077-1086, 2006.

20. Mah C, Byrne BJ and Flotte TR: Virus-based gene delivery systems. Clin Pharmacokinet 41: 901-911, 2002.

21. Carter PJ and Samulski RJ: Adeno-associated viral vectors as gene delivery vehicles. Int J Mol Med 6: 17-27, 2000.

22. Tu SP, Cui JT, Liston P, et al: Gene therapy for colon cancer by adeno-associated viral vector-mediated transfer of survivin Cys84Ala mutant. Gastroenterology 128: 361-375, 2005.

23. Tu SP, Jiang XH, Lin MC, et al: Suppression of survivin expression inhibits in vivo tumorigenicity and angiogenesis in gastric cancer. Cancer Res 63: 7724-7732, 2003.

24. Goldberg R: Oxaliplatin in colorectal cancer: current studies. Oncology 14 (Suppl. 11): S42-S47, 2000.

25. O'Connor DS, Schechner JS, Adida C, et al: Control of apoptosis during angiogenesis by survivin expression in endothelial cells. Am J Pathol 156: 393-398, 2000.

26. Mesri M, Morales-Ruiz M, Ackermann EJ, et al: Suppression of vascular endothelial growth factor-mediated endothelial cell protection by survivin targeting. Am J Pathol 158: 1757-1765, 2001

27. Wall NR, O'Connor DS, Plescia J, Pommier Y and Altieri DC: Suppression of survivin phosphorylation on Thr34 by flavopiridol enhances tumor cell apoptosis. Cancer Res 63: 230-235, 2003.

28. Grossman D, Kim PJ, Schechner JS and Altieri DC: Inhibition of melanoma tumor growth in vivo by survivin targeting. Proc Natl Acad Sci USA 98: 635-640, 2001.
29. Wang XF, Kerzerho J, Adotevi O, et al: Comprehensive analysis of HLA-DR- and HLA-DP4-restricted $\mathrm{CD}^{+}{ }^{+} \mathrm{T}$ cell response specific for the tumor-shared antigen survivin in healthy donors and cancer patients. J Immunol 181: 431-439, 2008.

30. Muchmore SW, Chen J, Jakob C, et al: Crystal structure and mutagenic analysis of the inhibitor-of-apoptosis protein survivin. Mol Cell 6: 173-182, 2000.

31. Pan L, Peng XC, Leng F, et al: Therapeutic effects of survivin dominant negative mutant in a mouse model of prostate cancer. J Cancer Res Clin Oncol: Mar 9, 2010 (Epub ahead of print).

32. Yu DD, Wang CT, Shi HS, et al: Enhancement of cisplatin sensitivity in lewis lung carcinoma by liposome-mediated delivery of a survivin mutant. J Exp Clin Cancer Res 29: 46, 2010.

33. Zhen HN, Zhang X, Hu PZ, et al: Survivin expression and its relation with proliferation, apoptosis, and angiogenesis in brain gliomas. Cancer 104: 2775-2783, 2005.

34. Tran J, Rak J, Sheehan C, et al: Marked induction of the IAP family antiapoptotic proteins survivin and XIAP by VEGF in vascular endothelial cells. Biochem Biophys Res Commun 264: 781-788, 1999

35. Tran J, Master Z, Yu JL, Rak J, Dumont DJ and Kerbel RS: A role for survivin in chemoresistance of endothelial cells mediated by VEGF. Proc Natl Acad Sci USA 99: 4349-4354, 2002.

36. Harfouche R, Hassessian HM, Guo Y, et al: Mechanisms which mediate the antiapoptotic effects of angiopoietin-1 on endothelial cells. Microvasc Res 64: 135-147, 2002.

37. Papapetropoulos A, Fulton D, Mahboubi K, et al: Angiopoietin-1 inhibits endothelial cell apoptosis via the Akt/survivin pathway. J Biol Chem 275: 9102-9105, 2000.

38. Zaffaroni N and Daidone MG: Survivin expression and resistance to anticancer treatments: perspectives for new therapeutic interventions. Drug Resist Updat 5: 65-72, 2002.

39. Haug K, Kravik KL and De Angelis PM: Cellular response to irinotecan in colon cancer cell lines showing differential response to 5-fluorouracil. Anticancer Res 28: 583-592, 2008.

40. William-Faltaos S, Rouillard D, Lechat P and Bastian G: Cell cycle arrest by oxaliplatin on cancer cells. Fundam Clin Pharmacol 21: 165-172, 2007.

41. William-Faltaos S, Rouillard D, Lechat P and Bastian G: Cell cycle arrest and apoptosis induced by oxaliplatin (L-OHP) on four human cancer cell lines. Anticancer Res 26: 2093-2099, 2006.

42. Liu HF, Hu HC and Chao JI: Oxaliplatin down-regulates survivin by p38 MAP kinase and proteasome in human colon cancer cells. Chem Biol Interact 188: 535-545, 2010. 